\title{
Minimizing SONET ADMs in unidirectional WDM rings with grooming ratio 3
}

\author{
Jean-Claude Bermond — Stephan Ceroi
}

$$
N^{\circ} 4626
$$

Novembre 2002

THÈME 1

\section{apport}

derecherche 



\title{
Minimizing SONET ADMs in unidirectional WDM rings with grooming ratio 3
}

\author{
Jean-Claude Bermond, Stephan Ceroi \\ Thème 1 - Réseaux et systèmes \\ Projet MASCOTTE
}

Rapport de recherche $\mathrm{n}^{\circ} 4626$ - Novembre 2002 - 10 pages

\begin{abstract}
We consider traffic grooming in WDM unidirectional rings with all-to-all uniform unitary traffic. We determine the minimum number of SONET/SDH add-drop multiplexers (ADMs) required when the grooming ratio is 3 . In fact, using tools of design theory, we solve the equivalent edge partitioning problem: find a partition of the edges of the complete graph on $n$ vertices $\left(K_{n}\right)$ into subgraphs having at most 3 edges and in which the total number of vertices has to be minimized.
\end{abstract}

Key-words: Traffic grooming, graph, edge-partition, design theory, WDM rings. 


\section{Minimisation du nombre d'ADM dans les anneaux WDM unidirectionnel avec un facteur de groupage 3}

Résumé : Nous considérons le problème du groupage de trafic dans les anneaux WDM unidirectionnels dans le cas d'un échange total avec un trafic unitaire uniforme. Nous déterminons le nombre minimum de multiplexeurs à insertion/extractions (ADM) lorsque le facteur de groupage est 3. En utilisant des outils de la théorie des designs, nous résolvons le problème équivalent de partition d'arêtes : trouver une partition des arêtes du graphe complet à $n$ sommets $\left(K_{n}\right)$ en sous-graphes ayant au plus 3 arêtes telle que le nombre total de sommets soit minimum.

Mots-clés : Groupage, graphe, partition des arêtes, théorie des designs, anneaux WDM. 


\section{Introduction}

Traffic grooming is the generic term for packing low rate signals into higher speed streams (see the surveys $[9,13,15]$ ). By using traffic grooming, one can bypass the electronics in the intermediate nodes. Typically, in a WDM network, instead of having one SONET/SDH Add Drop Multiplexer (or ADM) on every wavelength at every node, it may be possible to have ADMs only for the wavelengths used at that node (the other wavelengths being optically routed without electronic switching). The objective is either to minimize the transmission cost, in particular the number of wavelengths, or to minimize the equipment cost, in particular the total number of ADMs used in the network.

Here, we consider the particular case of unidirectional rings with static uniform symmetric all-to-all traffic. In this case, for each pair $\{i, j\}$, we associate a circle (or circuit) which contains both the request from $i$ to $j$ and from $j$ to $i$. If each circle requires only $\frac{1}{C}$ of the bandwidth of a wavelength, we can "groom" $C$ circles on the same wavelength. $C$ is called the grooming ratio (or grooming factor). For example, if the request from $i$ to $j$ (and from $j$ to $i$ ) is one OC-12 and a wavelength can carry an OC-48, the grooming factor is 4 . Given the grooming ratio $C$ and the size $n$ of the ring, the objective is to minimize the total number of ADMs used, denoted $A(C, n)$, and therefore to reduce the network cost by eliminating as many ADMs as possible from the "no grooming case". For example, let $n=4$; we have 6 circles corresponding to the 6 pairs $\{1,2\},\{1,3\},\{1,4\},\{2,3\},\{2,4\},\{3,4\}$. Without grooming, that is if we assign one wavelength per circle, we need $2 \mathrm{ADMs}$ per circle; then a total of $12 \mathrm{ADMs}$ are required. Suppose now that $C=3$, that is we can groom 3 circles on one wavelength. One can groom on wavelength 1 the circles associated with $\{1,2\},\{1,3\},\{1,4\}$ using $4 \mathrm{ADMs}$ and on wavelength 2 those associated with $\{2,3\},\{2,4\},\{3,4\}$ using 3 ADMs for a total of 7 ADMs.

This case of unidirectional rings with static uniform symmetric all-to-all traffic has been considered by many authors $[1,3,8,10,11,12,16,17,18,19,20]$ and numerical results, heuristics and tables have been given (see for example those in [17]). This case presents the advantage of concentrating on the grooming phase (excluding the routing). It can also be applied to groom components of more general connections than two opposite pairs into wavelengths or more general classes. These components are called circles [3, 20] or circuits [17] or primitive rings [6, 7].

In [1] it is noted that the problem of minimizing the number of ADMs for the unidirectional ring $C_{n}$, with a grooming factor $C$, can be expressed as follows: partition the edges of the complete graph on $n$ vertices $\left(K_{n}\right)$ into $W$ subgraphs $B_{\lambda}, \lambda=1,2, \ldots, W$, having $\left|E\left(B_{\lambda}\right)\right|$ edges and $\left|V\left(B_{\lambda}\right)\right|$ vertices, with $\left|E\left(B_{\lambda}\right)\right| \leq C$ and where $\sum_{\lambda=1}^{W}\left|V\left(B_{\lambda}\right)\right|$ has to be minimized (the edges of $K_{n}$ correspond to the circles, the subgraphs $B_{\lambda}$ correspond to the wavelengths and a vertex of $B_{\lambda}$ corresponds to an ADM). In [1] various results are given using tools of design theory [5] and they improve and unify all the preceding results in the

literature. Note that design theory was also used in $[6,7]$ for a slightly different problem with $C=8$, as they consider bidirectional rings and 4 requests are grouped in a circle. 
Here we completely solve the case $C=3$. This case is easy to solve when there exists a partition of $K_{n}$ into $K_{3}$ 's (triangles), as $K_{3}$ is the graph with 3 edges having the minimum number of vertices. For that we can use the existence of Steiner Triple Systems or $(n, 3,1)$ designs which can be stated as follows (for a proof see [2]).

Theorem 1 (Steiner's theorem) For any $n \equiv 1,3(\bmod 6)$, the edges of $K_{n}$ can be partitioned into $K_{3}$ 's.

Note that the problem we consider here is different from the problems of design theory in which one looks for a partition of the edges into isomorphic subgraphs and such a partition exists only for some values of $n$. For other values one can think to use results on packings or coverings to solve our problem. For example, for $C=3$ and $n \not \equiv 1$ or $3(\bmod 6)$, one can think that the best solution is obtained by taking as many $K_{3}$ 's as possible, but it does not necessarily lead to an optimal solution. Consider for example $K_{6}$. It can be partitioned into the 4 triangles $(1,2,3),(1,4,5),(2,4,6),(3,5,6)$ plus the 3 edges $1-6,2-5,3-4$. So, altogether we have 5 subgraphs and 18 ADMs. However, we can also partition $K_{6}$ into the $3 K_{3}$ 's $(1,2,3),(2,4,5),(3,5,6)$, the star $K_{1,3}$ with edges 1-4,1-5,1-6, and the path $P_{4}$ with edges 2-6,6-4,4-3. This solution uses 5 subgraphs and 17 ADMs. Similarly, if we use a covering of the edges of $K_{6}$ by $6 K_{3}$ 's and delete the edges covered twice, we will have to use 6 subgraphs with 3 vertices and altogether 18 vertices. More generally, when $n$ is even, a covering of $K_{n}$ by $K_{3}$ 's needs $\left[\frac{n^{2}}{6}\right]$ triangles and 3 times more ADMs; but we will show that the optimal solution uses about $\left\lceil\frac{n}{4}\right\rceil$ fewer ADMs.

Here, we determine the exact value of $A(3, n)$, denoted by $A(n)$. The main theorem can be stated as follows:

\section{Theorem 2}

(i) When $n$ is odd, $A(n)=n(n-1) / 2+\epsilon$, where $\epsilon=0$ if $n \equiv 1$ or $3(\bmod 6)$, and $\epsilon=2$ if $n \equiv 5(\bmod 6)$;

(ii) When $n$ is even, $A(n)=n(n-1) / 2+\left\lceil\frac{n}{4}\right\rceil+\epsilon$, where $\epsilon=1$ if $n \equiv 8(\bmod 12)$, and $\epsilon=0$ otherwise.

Furthermore our solution uses the minimum number of subgraphs (wavelengths) possible, that is $\left[\frac{n(n-1)}{6}\right]$; therefore for $C=3$, the conjecture of [3] that the minimum number of ADMs can be achieved with the minimum number of wavelengths is true (in [1] it is shown that the conjecture is false for many values of $C$, the first one being $C=7$ ).

\section{Notation}

As we mentioned in the introduction, we want to partition the edges of $K_{n}$ into subgraphs with at most 3 edges and to minimize the total number $A(n)$ of vertices in such a partition.

INRIA 
Here are the possible connected subgraphs with at most 3 edges:

\begin{tabular}{|l|l|l|l|l|}
\hline Name & Class & \# vertices & \# odd degree vertices \\
\hline$E$ & $\bullet$ & $\bullet$ & 2 & 2 \\
\hline$P_{3}$ & $\bullet$ & $\bullet$ & 3 & 2 \\
\hline$K_{3}$ & $\bullet$ & 0 & 3 & 0 \\
\hline$K_{1,3}$ & $\bullet$ & $\bullet$ & 4 & 4 \\
\hline$P_{4}$ & $\bullet$ & $\bullet$ & 4 & 2 \\
\hline
\end{tabular}

For a given partition $P$ of the edges of $K_{n}$, we denote by $a_{1}, a_{2}, a_{3}, b_{3}, c_{3}$ the number of subgraphs of type respectively $E, P_{3}, K_{3}, K_{1,3}, P_{4}$. By counting the number of edges of $K_{n}$ we have:

$$
a_{1}+2 a_{2}+3 a_{3}+3 b_{3}+3 c_{3}=n(n-1) / 2
$$

The sum of the number of vertices of the subgraphs in the partition $P$ is denoted $A(P)$. Thus

$$
A(P)=2 a_{1}+3 a_{2}+3 a_{3}+4 b_{3}+4 c_{3}=n(n-1) / 2+a_{1}+a_{2}+b_{3}+c_{3}
$$

Finally, following the definition of $A(n)$ given in the introduction, we have $A(n)=\min \left\{A(P): P\right.$ is a partition of $\left.K_{n}\right\}$.

\section{Lower bounds}

In this section we prove that $A(n)$ has at least the value given in Theorem 2. Let $P$ be any partition of the edges of $K_{n}$.

Case $(i)$ : if $n \equiv 1,3(\bmod 6)$, equation $(2)$ gives immediately $A(P) \geq n(n-1) / 2$. Suppose now that $n \equiv 5(\bmod 6)$. Thus $n(n-1) / 2 \equiv 1(\bmod 3)$. Then equation $(1)$ modulo 3 gives $a_{1}+2 a_{2} \neq 0$.

Suppose that $a_{1}+a_{2}+b_{3}+c_{3}=1$. Note that the subgraphs $E, P_{3}, K_{1,3}$ and $P_{4}$ have vertices with odd degree. As every vertex in $K_{n}$ has even degree, we have a contradiction, thus $a_{1}+a_{2}+b_{3}+c_{3} \geq 2$.

Then by equation $(2), A(P) \geq n(n-1) / 2+2$.

Case $(i i)$ : As $n$ is even, the degree of each vertex of $K_{n}$ is odd, so every vertex must be an odd degree vertex of at least one subgraph; but the number of odd degree vertices of $E, P_{3}, K_{3}, K_{1,3}$, and $P_{4}$ are 2,2,0,4,2 respectively; thus we have the following additional inequality:

$$
2 a_{1}+2 a_{2}+4 b_{3}+2 c_{3} \geq n
$$


From (3) we deduce that

$$
4\left(a_{1}+a_{2}+b_{3}+c_{3}\right) \geq n+2\left(a_{1}+a_{2}+c_{3}\right)
$$

So

$$
a_{1}+a_{2}+b_{3}+c_{3} \geq\left\lceil\frac{n}{4}\right\rceil
$$

which gives the result for the cases $n \not \equiv 8(\bmod 12)$.

Now if $n \equiv 8(\bmod 12)$, then $n(n-1) / 2 \equiv 1(\bmod 3)$ and thus equation (1) modulo 3 gives the additional constraint $a_{1}+2 a_{2} \geq 1$. Thus we have $4\left(a_{1}+a_{2}+b_{3}+c_{3}\right) \geq n+1$ and $A(P) \geq n(n-1) / 2+n / 4+1$ as required.

\section{Upper bounds}

Let $p_{1}, p_{2}, \ldots, p_{l}$ be some nonnegative integers; the complete multipartite graph with class sizes $p_{1}, p_{2}, \ldots, p_{l}$, denoted $K_{p_{1}, p_{2}, \ldots, p_{l}}$ is defined to be the graph with vertex set $P_{1} \cup P_{2} \cup$ $\ldots \cup P_{l}$ where $\left|P_{i}\right|=p_{i}$, and two vertices $x \in P_{i}$ and $y \in P_{j}$ are adjacent if and only if $i \neq j$. For $t>0$, we denote $K_{g \times t}$ (resp. $\left.K_{g \times t, u}\right)$ by $K_{g, g, \ldots, g}\left(\right.$ resp. $\left.K_{g, g, \ldots, g, u}\right)$ where $g$ occurs $t$ times.

Using terminology of design theory, the existence of a partition of the edges of $K_{p_{1}, p_{2}, \ldots, p_{l}}$ into $K_{k}$ is equivalent to the existence of a $k-G D D$ (group divisible design) with group sizes $p_{1}, p_{2}, \ldots, p_{l}$, also known as $k-G D D$ of type $g_{1}^{a_{1}} g_{2}^{a_{2}} \ldots g_{s}^{a_{s}}$, where there are $a_{i}$ values of the $p_{j}$ 's equal to $g_{i}$.

The following theorem of Colbourn [4] (see also [14, Theorem 1.24]) will be used repeatedly:

Theorem 3 Let $g, t$ and $u$ some nonnegative integers. $K_{g \times t, u}$ can be decomposed into $K_{3}$ 's if and only if the following conditions are all satisfied:

(i) if $g>0$ then $t \geq 3$, or $t=2$ and $u=g$, or $t=1$ and $u=0$, or $t=0$;

(ii) $u \leq g(t-1)$ or $g t=0$;

(iii) $g(t-1)+u \equiv 0(\bmod 2)$ or $g t=0$;

(iv) $g t \equiv 0(\bmod 2)$ or $u=0$;

(v) $g^{2} t(t-1) / 2+g t u \equiv 0(\bmod 3)$.

We now prove the upper bounds in Theorem 2. Actually we give a stronger result, by exhibiting the classes of the decomposition. 
Theorem 4 Let $n \geq 2$. There exists a partition of $K_{n}$ using

1. if $n \equiv 1,3(\bmod 6), \frac{n(n-1)}{6} K_{3}$;

2. if $n \equiv 5(\bmod 6), \frac{n(n-1)-8}{6} K_{3}$ and $2 P_{3}$;

3. if $n \equiv 0,4(\bmod 12), \frac{n(n-1)}{6}-\frac{n}{4} K_{3}$ and $\frac{n}{4} K_{1,3}$;

4. if $n \equiv 2,8(\bmod 12), \frac{n(n-1)-2}{6}-\left\lceil\frac{n-2}{4}\right\rceil K_{3},\left\lceil\frac{n-2}{4}\right\rceil K_{1,3}$ and $1 E$;

5. if $n \equiv 6,10(\bmod 12), \frac{n(n-1)}{6}-\frac{n+2}{4} K_{3}, \frac{n-2}{4} K_{1,3}$ and $1 P_{4}$.

\section{Proof.}

First case $: n$ odd.

If $n \equiv 1,3(\bmod 6)$, the result is exactly Theorem 1. Suppose now that $n \equiv 5(\bmod 6)$.

First we deal with the cases $n=5$ and $n=11$ :

- $K_{5}$ can be decomposed into $2 K_{3}$ 's $(1,2,3),(1,4,5)$, and $2 P_{3}$ 's with edges $4-2,2-5$ and $4-3,3-5$;

- $K_{11}$ is the union of a $K_{5}$ and a $K_{1 \times 6,5}$. This $K_{5}$ can be decomposed as seen above into $2 K_{3}$ 's and $2 P_{3}$ 's. By Theorem 3 (with $g=1, t=6$ and $u=5$ ), $K_{1 \times 6,5}$ can be decomposed into $15 K_{3}$ 's. So $K_{11}$ can be decomposed into $17 K_{3}$ 's and $2 P_{3}$ 's.

Now for $n \geq 17$ and $n=6 p+5(p \geq 2), K_{6 p+5}$ is the union of $2 p K_{3}$ 's, $1 K_{5}$ and a $K_{3 \times 2 p, 5}$. But $K_{5}$ can be decomposed into $2 K_{3}$ 's and $2 P_{3}$ 's, and $K_{3 \times 2 p, 5}$ can be decomposed into $K_{3}$ 's by Theorem 3 with $g=3, t=2 p$ and $u=5$.

\section{Second case : $n$ even.}

First, we deal with the following small cases:

- case $n=2$ : trivial;

- case $n=4: K_{4}$ can be decomposed into the $K_{3}(1,2,3)$ and the $K_{1,3}$ with edges $4-1,4-2,4-3$

- case $n=6$ : as stated in the introduction, $K_{6}$ can be decomposed into the $P_{4}$ with edges $2-6,6-4,4-3$, the $K_{1,3}$ with edges $1-4,1-5,1-6$, and the $K_{3}$ 's $(1,2,3),(2,4,5),(3,5,6)$;

- case $n=8:$ take a decomposition of $K_{7}$ in $K_{3}$ 's, and connect an additional vertex $\infty$ to all vertices of the $K_{7}$. The 7 edges incident to $\infty$ can be decomposed in $2 K_{1,3}$ 's and one $E$; 
- case $n=10: K_{10}$ can be decomposed into the $K_{1,3}$ with edges 9-2,9-4,9-6, the $K_{1,3}$ with edges $10-1,10-3,10-5$, the $P_{4}$ with edges 8-9,9-10,10-7, and the following $K_{3}$ 's:

$\begin{array}{ccc}(1,5,6) & (1,2,8) & (1,3,9) \\ (2,6,7) & (2,3,5) & (2,4,10) \\ (3,7,8) & (3,4,6) & (5,7,9) \\ (4,8,5) & (4,1,7) & (6,8,10)\end{array}$

Now let $n=4 t+u$ with $t \equiv 0(\bmod 3), t \geq 3$ and $u=0,2,4,6,8$ or $10 . K_{n}$ can be decomposed into $t K_{4}$ 's, a $K_{u}$ and a $K_{4 \times t, u}$. By Theorem 3 with $g=4, K_{4 \times t, u}$ can be decomposed into $K_{3}$ 's, except when $t=3$ and $u=10$, i.e. $n=22$, for which the condition (ii) is not satisfied. Now for $n \neq 22$, each $K_{4}$ can be decomposed into a $K_{3}$ and a $K_{1,3}$, and $K_{u}$ can be decomposed as shown above. Hence we get a partition of $K_{n}$ into the required number of $K_{3}$ 's, $K_{1,3}$ 's, $E$ and $P_{4}$.

Finally for $n=22, K_{22}$ can be decomposed into $4 K_{4}$ 's, a $K_{6}$ and a $K_{4 \times 4,6}$. Each $K_{4}$ can be decomposed into a $K_{3}$ and a $K_{1,3}$. The $K_{6}$ can be decomposed as seen above into a $P_{4}$, a $K_{1,3}$, and $3 K_{3}$ 's. By Theorem 3 with $g=t=4$ and $u=6, K_{4 \times 4,6}$ can be decomposed into $K_{3}$ 's. Thus we get a partition of $K_{22}$ into a $P_{4}, 5 K_{1,3}$ 's and some $K_{3}$ 's, as required.

Note that, as mentioned in the introduction, our solution uses the minimum number of subgraphs possible, that is $\left[\frac{n(n-1)}{6}\right]$, showing that for $C=3$, the conjecture of [3] is true.

\section{Conclusion}

In this article, we have determined the minimum number of SONET add-drop multiplexers (ADMs) required with a grooming ratio 3 in unidirectional WDM rings with all-to-all uniform unitary traffic. We have also shown that this minimum number is attained with a minimum number of wavelengths. The same ideas can be used to determine the minimum number $A(C, n)$ for larger values of $C$. For $C=4$ an optimal solution can be obtained easily as we can partition the edges of $K_{n}$ into $C_{4}$ 's and $K_{3}+E$. Therefore we have $A(4, n)=n(n-1) / 2$ with the minimum number of wavelengths, a result obtained also in [12]. We have also obtained partial results for the cases $C=5$ and $C=6$. In the latter case we can use results on 4-GDD but they are not sufficient for all the congruence classes.

Acknowledgment. We thank David Coudert and Joseph Yu for their helpful comments.

\section{References}

[1] J.-C. Bermond and D. Coudert. Uniform all-to-all traffic grooming in unidirectional WDM ring networks. Submitted to IEEE International Conference on Communications - ICC'03. 
[2] Peter J. Cameron. Combinatorics: topics, techniques, chapter 8: Steiner triple systems. Cambridge University Press, Cambridge, England, 1994.

[3] A. L. Chiu and E. H. Modiano. Traffic grooming algorithms for reducing electronic multiplexing costs in WDM ring networks. IEEE/OSA Journal of Lightwave Technology, 18 (2000), 2-12.

[4] C.J. Colbourn. Small group divisible designs with block size 3. J. Combin. Math Combin. Comput., 14 (1993), 151-171.

[5] C.J. Colbourn and J. Dinitz, editors. The CRC handbook of Combinatorial designs. CRC Press, New-York, 1996.

[6] C.J. Colbourn and A. Ling. Wavelength add-drop multiplexing for minimizing SONET ADMs. Discrete Applied Mathematics, to appear.

[7] C.J. Colbourn and P-J. Wan. Minimizing drop cost for SONET/WDM networks with $\frac{1}{8}$ wavelength requirements. Networks, 37 (2001), 107-116.

[8] R. Dutta and N. Rouskas. On optimal traffic grooming in WDM rings. IEEE Journal of Selected Areas in Communications, 20 (2002), 1-12.

[9] R. Dutta and N. Rouskas. Traffic grooming in WDM networks: Past and future. Technical report, CSC TR-2002-08, NCSU, 2002.

[10] O. Gerstel, P. Lin, and G. Sasaki. Wavelength assignment in a WDM ring to minimize cost of embedded SONET rings. In IEEE Infocom, pages 94-101, San Francisco, California, 1998.

[11] O. Gerstel, R. Ramaswani, and G. Sasaki. Cost-effective traffic grooming in WDM rings. IEEE/ACM Transactions on Networking, 8 (2000), 618-630.

[12] J.Q. Hu. Optimal traffic grooming for wavelength-division-multiplexing rings with allto-all uniform traffic. OSA Journal of Optical Networks, 1 (2002), 32-42.

[13] E. Modiano and P. Lin. Traffic grooming in WDM networks. IEEE Communications Magazine, 39 (2001), 124-129.

[14] Ronald C. Mullin and Hans-Dietrich O.F. Gronau. CRC handbook of Combinatorial designs, chapter III : PBDs and GDDs : The Basics. CRC Press, New-York, 1996.

[15] A. Somani. Survivable traffic grooming in WDM networks. In D.K. Gautam, editor, Broad band optical fiber communications technology - BBOFCT, pages 17-45, Jalgaon, India, December 2001. Nirtali Prakashan. Invited paper.

[16] P-J. Wan, G. Calinescu, L. Liu, and O. Frieder. Grooming of arbitrary traffic in SONET/WDM BLSRs. IEEE Journal of Selected Areas in Communications, 18 (2000), 1995-2003.

$\mathrm{RR} \quad \mathrm{n}^{\circ} 4626$ 
[17] J. Wang, W. Cho, V. Vemuri, and B. Mukherjee. Improved approches for cost-effective traffic grooming in WDM ring networks: Ilp formulations and single-hop and multihop connections. IEEE/OSA Journal of Lightwave Technology, 19 (2001), 1645-1653.

[18] X. Yuan and A. Fulay. Wavelength assignment to minimize the number of SONET ADMs in WDM rings. In IEEE International Conference on Communications - ICC, New York, April 2002.

[19] X. Zhang and C. Qiao. On optimal all-to-all personalized connections and cost-effective designs in WDM rings. IEEE/ACM Transactions on Networking, 7 (1996), 435-445.

[20] X. Zhang and C. Qiao. An effective and comprehensive approach for traffic grooming and wavelength assignment in SONET/WDM rings. IEEE/ACM Transactions on Networking, 8 (2000), 608-617. 


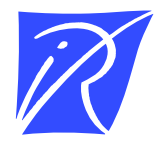

Unité de recherche INRIA Sophia Antipolis 2004, route des Lucioles - BP 93 - 06902 Sophia Antipolis Cedex (France)

Unité de recherche INRIA Lorraine : LORIA, Technopôle de Nancy-Brabois - Campus scientifique 615, rue du Jardin Botanique - BP 101 - 54602 Villers-lès-Nancy Cedex (France)

Unité de recherche INRIA Rennes : IRISA, Campus universitaire de Beaulieu - 35042 Rennes Cedex (France)

Unité de recherche INRIA Rhône-Alpes : 655, avenue de l'Europe - 38330 Montbonnot-St-Martin (France)

Unité de recherche INRIA Rocquencourt : Domaine de Voluceau - Rocquencourt - BP 105 - 78153 Le Chesnay Cedex (France)

Éditeur

INRIA - Domaine de Voluceau - Rocquencourt, BP 105 - 78153 Le Chesnay Cedex (France)

http://www.inria.fr

ISSN 0249-6399 